\title{
Retrospective assessment of patient characteristics and healthcare costs prior to a diagnosis of Alzheimer's disease in an administrative claims database
}

Radhika Nair ${ }^{1}$, Virginia S. Haynes ${ }^{2,4^{*}}$, Mir Siadaty ${ }^{1}$, Nick C. Patel ${ }^{1}$, Adam S. Fleisher ${ }^{2}$, Derek Van Amerongen ${ }^{3}$, Michael M. Witte ${ }^{2}$, AnnCatherine M. Downing ${ }^{2}$, Leslie Ann Hazel Fernandez', Vishal Saundankar ${ }^{1}$ and Daniel E. Ball ${ }^{2}$

\begin{abstract}
Background: The objective of this study was to examine patient characteristics and health care resource utilization $(\mathrm{HCRU})$ in the 36 months prior to a confirmatory diagnosis of Alzheimer's disease (AD) compared to a matched cohort without dementia during the same time interval.

Methods: Patients newly diagnosed with AD (with $\geq 2$ claims) were identified between January 1, 2013 to September 31,2015 , and the date of the second claim for AD was defined as the index date. Patients were enrolled for at least 36 months prior to index date. The AD cohort was matched to a cohort with no AD or dementia codes (1:3) on age, gender, race/ethnicity, and enrollment duration prior to the index date. Descriptive analyses were used to summarize patient characteristics, HCRU, and healthcare costs prior to the confirmatory AD diagnosis. The classification and regression tree analysis and logistic regression were used to identify factors associated with the AD diagnosis.
\end{abstract}

Results: The AD cohort $(N=16,494)$ had significantly higher comorbidity indices and greater odds of comorbid mental and behavioral diagnoses, including mild cognitive impairment, mood and anxiety disorders, behavioral disturbances, and cerebrovascular disease, heart disease, urinary tract infections, and pneumonia than the matched non-AD or dementia cohort $(N=49,482)$. During the six-month period before the confirmatory AD diagnosis, AD medication use and diagnosis of mild cognitive impairment, Parkinson's disease, or mood disorder were the strongest predictors of a subsequent confirmatory diagnosis of AD. Greater HCRU and healthcare costs were observed for the AD cohort primarily during the six-month period before the confirmatory AD diagnosis.

Conclusion: The results of this study demonstrated a higher comorbidity burden and higher costs for patients prior to a diagnosis of $\mathrm{AD}$ in comparison to the matched cohort. Several comorbidities were associated with a subsequent diagnosis of AD.

Keywords: Alzheimer's disease, Comorbidities, Costs

\footnotetext{
* Correspondence: ginger.haynes@lilly.com

Eli Lilly and Company, Indianapolis, USA

${ }^{4}$ Lilly Corporate Center, Drop Code 1730, Indianapolis, IN 46285, USA

Full list of author information is available at the end of the article
}

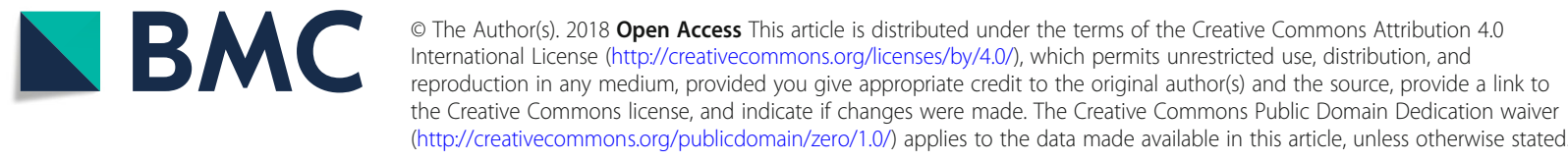




\section{Background}

Development of Alzheimer's disease (AD) occurs more frequently in the elderly. With an increasing elderly population in the U.S., the prevalence and associated healthcare costs of $\mathrm{AD}$ are expected to rise significantly in the absence of any intervention or medication to slow or stop cognitive and functional decline in these patients [1]. Many of the compounds in development to address this issue aim to target the underlying AD pathophysiology, such as modulation of amyloid and tau protein deposition, and therefore may have the potential to slow the progression of disease. In anticipation of this potential shift away from the treatment paradigm of the currently available AD-indicated medications, which are mainly used for symptom management, the ability to accurately identify individuals earlier in the course of disease, prior to irreversible neuronal dysfunction, becomes critical [2, 3]. Therefore, clinical tools are needed to help identify patients earlier in the spectrum of illness and facilitate the ongoing development of disease modifying agents with the ability to alter AD progression.

For research studies that utilize administrative claims data, current diagnostic codes make it challenging to identify patients with $\mathrm{AD}$ until the later stages of the illness and require unique approaches to study trends before diagnosis occurs. Prior studies have used various methods to delineate how patients with $\mathrm{AD}$ are identified. These include, prospective observational cohorts, [4] using samples derived from electronic medical records of family practitioners, [5] Medicare databases, [6] Medicaid databases, [7] and commercial managed healthcare databases [8-10].

Among the studies utilizing administrative data, Gilden et al. [6] identified four major pathways that led to an AD diagnosis among Medicare Fee For Service (FFS) beneficiaries: AD as the initial diagnosis or cognitive disturbance followed by $\mathrm{AD}$; dementia with suspected etiologies, followed by $\mathrm{AD}$; dementia without known cause, followed by $\mathrm{AD}$; and a triple pathway which included cognitive disturbance followed by dementia of unknown cause, followed by AD. Jaakkimainen et al. [5] described an algorithm of "one hospitalization code or three physician claims codes at least 30 days apart in a two year period OR a prescription filled for an Alzheimer's disease and related dementias (AD-RD) specific medication" with high sensitivity, specificity, and positive predictive value to identify AD-RD. These studies highlight the challenge of identifying patients with AD from administrative claims data.

Along with the identification of patients with $\mathrm{AD}$, several studies have aimed to characterize the management of these patients prior to diagnosis. In the 12-18 month period prior to the initial diagnosis of $\mathrm{AD}$, patients tend to have increases in healthcare resource utilization (HCRU) and healthcare costs $[6,9,11]$. A study by Gilden et al. (2015)
[6] demonstrated a rapid increase in total monthly Medicare expenditures shortly before $\mathrm{AD}$ diagnosis, followed by a rapid decline in expenditures. In both Medicare and Medicaid populations, individuals with $\mathrm{AD}$ or $\mathrm{AD}-\mathrm{RD}$ incurred higher expenditures than matched controls in the 12 months prior to diagnosis $[7,9,11]$. A majority of these increases have been attributed to outpatient services, inpatient, and acute care services $[7,11]$.

The objective of the current study was to understand the pre-diagnostic journey of a cohort of patients who were newly diagnosed with $\mathrm{AD}$. The characterization of this pre-diagnostic journey of patients with AD builds on previous research through the examination of clinical characteristics, socioeconomic attributes, and behavioral characteristics. In addition, this study captures HCRU and costs during the 36 months prior to a confirmatory $\mathrm{AD}$ diagnosis in comparison to a matched cohort without AD or dementia. With a longer window of examination prior to a confirmed diagnosis of $\mathrm{AD}$, the current study also aimed to identify potential indicators available in administrative claims data that may help to predict patients who will be subsequently diagnosed with AD.

\section{Methods}

This was a retrospective, observational study using a linked database comprised of information from two databases, retrospective claims and the AmeriLINK data provided by Knowledge Base Management (KBM). The claims data includes billing for inpatient and outpatient office visits and outpatient prescription medication filled for millions of participants. The data includes claims for patients enrolled in commercial or Medicare plans. For the purposes of this study, we included individuals enrolled in commercial or Medicare Advantage and Prescription Drug plans (MAPD) with both medical and pharmacy coverage. Medicare Advantage plans are insurance plans offered to consumers through private companies that cover medical and hospital services that are included under Medicare parts A and B and include additional coverage not available in Medicare, typically including a prescription drug plan. The US federal government reimburses private companies approved to sell Medicare Advantage plans for those services covered in Medicare parts A and B. The consumer's premium covers additional services and benefits that are unique to the Medicare Advantage Plan [12].

All medical and pharmacy claims included in the study are fully adjudicated and paid. The enrollment, medical, and pharmacy claims data of individuals enrolled in the MAPD were linked using a unique identifier to three variables from AmeriLINK data from KBM. AmeriLINK data consists of consumer, census, and computed behavioral data using publicly available information (i.e., public records), retail transaction data (i.e., credit card purchases), 
and computed variables derived from census-type information and/or the combination of data to generate new variables. For this study we included three variables from AmeriLINK namely, estimated household income, percent 2010 white collar and blue collar employed (the percentage of the population in the census-area employed in a white collar or blue collar industry). The study protocol that included a description of the research database and methods was reviewed and approved by an external institutional review board.

Patients 55-89 years of age with two or more claims for AD [International Statistical Classification of Diseases, Ninth Revision, Clinical Modification (ICD-9-CM) $331.0 \times$ ] on different dates within 18 months of each other between January 1, 2013 and September 30, 2015, were identified (Fig. 1). The date of the second claim for $\mathrm{AD}$ was set as the index date. Patients were required to have continuous enrollment for at least 36 months prior to the index date, and had no medical claims with diagnosis codes for $\mathrm{AD}$ during the pre-index period (with the exception of the first medical claim for AD).

This AD cohort was matched at the individual patient level with a cohort during the same time interval with no $\mathrm{AD}$ or dementia codes (matched cohort) on age (same age in years), gender, race/ethnicity, and enrollment duration prior to the index date ( \pm 3 months). Each individual patient in the $\mathrm{AD}$ cohort was matched based on demographics with three individual patients from the control group of patients without any $\mathrm{AD}$ or dementia codes. The cohorts were matched only on demographics to allow for a clearer evaluation of the patient's clinical characteristics prior to the diagnosis of $\mathrm{AD}$, while minimizing any demographic differences.

$\mathrm{AD}$ is a form of dementia; therefore, it is likely that patients with a diagnosis of dementia, might actually have AD. To ensure our matched cohort did not have undiagnosed $\mathrm{AD}$ dementia, we required that they have no diagnosis codes for dementia. Conversely, for the AD cohort, it is possible that physicians would document dementia without confidence in classifying as $\mathrm{AD}$, so we did not want to

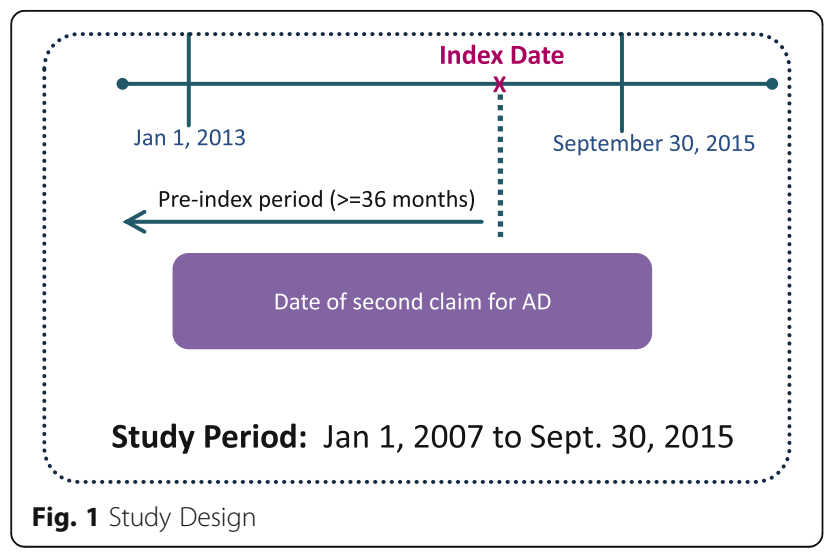

exclude patients with two diagnoses of $\mathrm{AD}$ dementia due to the presence of a less specific dementia code.

This matched non-AD or dementia cohort were required to have a medical claim within 30 days of the index date of the matched patient from the $\mathrm{AD}$ cohort and no medical claims with diagnosis codes for $\mathrm{AD}$, mild cognitive impairment (MCI; ICD-9-CM: 331.83), or $\mathrm{AD}$-related or unrelated dementia codes for the entire length of enrollment. The index date for the matched patient was the date of their medical claim which was within 30 days of their matched AD cohort member's second diagnosis.

\section{Variables}

Medical and pharmacy claims data, along with AmeriLINK data, were examined prior to the confirmatory $\mathrm{AD}$ diagnosis in order to understand demographic, clinical, and socioeconomic/behavioral characteristics of these patients. Prior to the confirmatory diagnosis of $A D$, baseline demographics including age, gender, race/ethnicity (MAPD only), region of residence, insurance type (MAPD or commercial), and low income subsidy status were evaluated. Low income subsidy status refers to patients with limited resources and an income below 150\% of the U.S. federal poverty threshold who were eligible for additional premium and cost-share assistance for prescription drugs. Patients eligible for both Medicare and Medicaid (dual eligible) were also identified.

Comorbidities were evaluated using the Deyo Charlson Comorbidity Index (DCCI) score [13-15] and the RxRisk-V Score [16]. The DCCI score is based on 17 categories of comorbidities, which are used to calculate a score that reflects the cumulative increased likelihood of one-year mortality [13]. The evolution of the Deyo-Charlson methodology has permitted researchers to use the score as an assessment of overall patient health risk. The RxRisk-V Score [16] is a pharmacy-based comorbidity index that involves the identification of 45 distinct medical condition categories via their associated medication treatments. Of the 45 conditions, three that are defined based on claims for durable medical equipment (neurogenic bladder, ostomy, and urinary incontinence) were not included in this study given these claims are not captured in pharmacy claims data.

The prevalence of pre-specified chronic comorbidities and the proportions of patients with annual wellness visits and cognitive assessments prior to the diagnosis of $\mathrm{AD}$ were compared between the AD cohort and the matched cohort. The proportion of patients who filled prescriptions for $\mathrm{AD}$ medications [cholinesterase inhibitors (donepezil, galantamine, rivastigmine) or $\mathrm{N}$-methyl-D-aspartate (NMDA) antagonist (memantine)] during the pre-index period was also compared across both cohorts.

To evaluate trends in utilization, HCRU and healthcare costs were measured at 6-month intervals prior to 
the confirmatory diagnosis of AD. Medical HCRU included the number of outpatient, inpatient, and emergency department (ED) visits. Pharmacy HCRU included the calculated number of unique medication classes filled by a patient. The total costs (paid by plan and patient) included medical (outpatient, inpatient, and ED), pharmacy, and total healthcare costs (medical plus pharmacy); these costs were adjusted to 2015.

Socioeconomic and behavioral characteristics play an important role in patient behavior related to the consumption of health care. As such, we used variables identified from the AmeriLINK data including household income, occupation in the census area where the patient resided (blue collar vs. white collar), and education. Furthermore, we used behavior segmentation developed by Humana that provides insights on how individuals naturally group themselves based on their general propensity to engage in their health/healthcare system and with the health plan. The behavior segmentation includes multiple distinct behavioral groups, and are further classified into whether individuals have chronic health issues (chronic) or not (healthy). At the time of this study, the behavior segmentation was applied only to the MAPD population; however, the number of patients in this study enrolled in a commercial plan was low (1.0\%). The behavior segmentation was included in the analyses to control for individual variations, but the results for the segments themselves are not provided due to their proprietary nature.

\section{Analyses}

Descriptive analyses were used to compare the pre-index demographic, clinical, and socioeconomic/behavioral characteristics of the $\mathrm{AD}$ and matched cohorts. HCRU and healthcare costs for the $0-36$ months prior to the index date were analyzed for both cohorts. Mean and median cost components were computed, and mean cost components were compared across groups using t-test.

The associations between patient characteristics and $\mathrm{AD}$ diagnosis were tested using multivariate logistic regression models. Given the large number of variables and uncertainty as to their potential interactions, a classification and regression tree (CART) $[17,18]$ method was used to refine the selection of variables for inclusion in the logistic regression model. The CART analysis is a decision tree method that uses recursive partitioning of data into strata, enabling a study population to be categorized into meaningful subsets. The advantages of using CART are that it is a non-parametric technique not dependent on assumptions regarding distribution of the variables in a dataset, and it can be used to assess both dichotomous and continuous outcome variables [18].

In addition to the selection of variables for inclusion, the CART analysis further aided in identifying the most important explanatory variables in the dataset, which were most predictive of a diagnosis of $\mathrm{AD}$. The top 15 explanatory variables from the CART analysis, along with their two-way interactions, were included in the logistic regression models.

For the final logistic regression model, the forward selection method was used with entry criteria of $P \leq 0.05$ and retention criteria of $P \leq 0.2$. In the results for the regression analysis, the ratios of odds ratios (RORs) are reported for interactions. The RORs is an estimate derived from dividing one odds ratio (OR) by another (i.e., OR X/ OR Y) when calculating the interaction of one factor with another. In the logistic regression models, the ROR is the exponent of the beta of the two-way interaction term. For example, variable $\mathrm{X}$ has $\mathrm{OR}$ of 50 and variable $\mathrm{Y}$ has $\mathrm{OR}$ of 100; both ORs are larger than the neutral value of 1 . The ratio of OR X/OR Y is $50 / 100$, or 0.5 ; this ROR is smaller than 1; however, having an ROR less than 1 does not mean the interaction of variables $\mathrm{X}$ and $\mathrm{Y}$ has led to a decrease in the odds of having the outcome.

\section{Results}

A total of 16,558 patients newly diagnosed with $\mathrm{AD}$ were identified, and of these, 16,494 patients were matched with 49,482 patients in the matched cohort (with no AD or dementia-related diagnoses). Patients in both cohorts were 79.9 [standard deviation (SD) 6.1] years old, with a higher proportion of women (59.4\%), and of white race (84.1\%, Table 1$)$. The majority of patients were enrolled in MAPD ( 99\%), and the average length of pre-index enrollment was 68.9 (SD 19.4) months.

Sociodemographically (Table 1), the AD cohort was from communities with fewer white-collar, employed professionals. Based on behavior segmentation, a larger proportion of the $\mathrm{AD}$ cohort was classified as having chronic disease $(53.3 \%$ vs. $37.1 \%)$, compared with the matched cohort, and the patterns of behavior regarding their healthcare varied within the classifications of Healthy and Chronic. An index diagnosis for AD was made by the primary care physician in $35.1 \%$ of the patients and $77.5 \%$ of patients had multiple visits to the index physician prior to index date. The average time between the two claims for $\mathrm{AD}$ was 92 [SD 125] days.

A significantly higher proportion of the matched cohort had a claim for an annual wellness visit compared to the AD cohort $(35.0 \%$ vs. $29.5 \%, P<0.001$, Table 2$)$, but a greater proportion of the AD cohort had cognitive assessments (2.6\% vs. $0.2 \%, P<0.001$, Table 2$)$. The majority of patients in the $\mathrm{AD}$ cohort (64.0\%) had filled a prescription for at least one $\mathrm{AD}$ medication during the pre-index period.

The AD cohort had significantly higher DCCI scores (3.8 vs. $3.0, P<0.001)$ and RxRisk-V Scores (9.8 vs. 8.3, $P<0.001$ ) than the matched cohort (Table 2), indicating a higher comorbidity burden. Among the pre-specified 
Table 1 Demographic, socioeconomic and behavioral characteristics of Alzheimer's disease and matched cohorts

\begin{tabular}{|c|c|c|c|}
\hline Characteristic & $A D$ & Matched Cohort (no AD or dementia) & $P$ value \\
\hline $\mathrm{N}$ & 16,494 & 49,482 & - \\
\hline Age in years, mean [SD] & $79.9[6.1]$ & $79.9[6.1]$ & 0.830 \\
\hline \multicolumn{4}{|l|}{ Gender, n (\%) } \\
\hline Female & $9800(59.4)$ & $29,400(59.4)$ & \multirow[t]{2}{*}{1.000} \\
\hline Male & $6694(40.6)$ & $20,082(40.6)$ & \\
\hline \multicolumn{4}{|l|}{ Race/ Ethnicity, n (\%) } \\
\hline White & $13,879(84.1)$ & $41,637(84.1)$ & \multirow[t]{4}{*}{1.000} \\
\hline Black & $1970(11.9)$ & $5910(11.9)$ & \\
\hline Hispanic & $317(1.9)$ & $951(1.9)$ & \\
\hline Other & $328(1.9)$ & $984(1.9)$ & \\
\hline \multicolumn{4}{|l|}{ Geographic Region, n (\%) } \\
\hline Northeast & $328(1.9)$ & $1081(2.2)$ & \multirow[t]{4}{*}{$<0.001$} \\
\hline Midwest & $4080(24.7)$ & $13,227(26.7)$ & \\
\hline South & $10,859(65.8)$ & $30,862(62.4)$ & \\
\hline West & $1227(7.4)$ & $4312(8.7)$ & \\
\hline \multicolumn{4}{|l|}{ Plan Type, n (\%) } \\
\hline MAPD & $16,391(99.4)$ & $48,923(98.9)$ & \multirow[t]{2}{*}{$<0.001$} \\
\hline Commercial & $103(0.6)$ & $559(1.1)$ & \\
\hline \multicolumn{4}{|l|}{ Plan Characteristics (MAPD), n (\%) } \\
\hline Low Income Subsidy Status Only & $2566(15.5)$ & $4531(9.1)$ & $<0.001$ \\
\hline Dual Eligibility (Medicare and Medicaid) Only & $32(0.2)$ & $61(0.1)$ & 0.030 \\
\hline Length of Pre-index Enrollment - mean [SD] & $68.9[19.4]$ & $68.9[19.4]$ & 0.980 \\
\hline \multicolumn{4}{|l|}{ Estimated Household Income, n (\%) } \\
\hline$<\$ 15,000$ & $3465(21.0)$ & $10,748(21.7)$ & \multirow[t]{6}{*}{0.003} \\
\hline$\$ 15,000-\$ 29,999$ & $2589(15.7)$ & $7948(16.0)$ & \\
\hline$\$ 30,000-\$ 49,999$ & $3433(20.8)$ & $10,467(21.1)$ & \\
\hline$\$ 50,000-\$ 99,999$ & $3749(22.7)$ & $12,250(24.7)$ & \\
\hline$>=\$ 100,000^{¥}$ & $1084(6.6)$ & $3757(7.6)$ & \\
\hline Unknown & $2174(13.2)$ & $4312(8.7)$ & \\
\hline \multicolumn{4}{|l|}{ Census 2010 Percent Blue Collar Employed, n (\%) } \\
\hline $0-14 \%$ & $2940(17.8)$ & $10,086(20.3)$ & \multirow[t]{6}{*}{$<0.001$} \\
\hline $15-20 \%$ & $2802(16.9)$ & $9361(18.9)$ & \\
\hline $21-26 \%$ & 3077 (18.6) & $9765(19.7)$ & \\
\hline $27-33 \%$ & $3030(18.4)$ & $8846(17.8)$ & \\
\hline $34-99 \%$ & $2471(14.9)$ & $7112(14.3)$ & \\
\hline Unknown & $2174(13.2)$ & $4312(8.7)$ & \\
\hline \multicolumn{3}{|l|}{ Census 2010 Percent White Collar Employed, n (\%) } & \multirow[t]{7}{*}{$<0.001$} \\
\hline $0-48 \%$ & $3149(19.1)$ & $9305(18.8)$ & \\
\hline $49-56 \%$ & $3167(19.2)$ & $9505(19.2)$ & \\
\hline $57-64 \%$ & $2981(18.1)$ & $9620(19.4)$ & \\
\hline $65-74 \%$ & $2875(17.4)$ & $9406(19.0)$ & \\
\hline $75-99 \%$ & $2148(13.0)$ & $7334(14.8)$ & \\
\hline Unknown & 2174 (13.2) & 4312 (8.7) & \\
\hline
\end{tabular}


Table 1 Demographic, socioeconomic and behavioral characteristics of Alzheimer's disease and matched cohorts (Continued)

\begin{tabular}{|c|c|c|c|}
\hline Characteristic & $A D$ & Matched Cohort (no AD or dementia) & $P$ value* \\
\hline \multicolumn{4}{|l|}{ Index AD diagnosing Provider, $\mathrm{n}(\%)(N=16,558)$} \\
\hline Primary Care & $5800(35.1)$ & & \\
\hline Geriatrics & $526(3.2)$ & & \\
\hline Neurology & $2225(13.5)$ & & \\
\hline Psychiatry & $813(4.9)$ & & \\
\hline Psychology & $240(1.5)$ & & \\
\hline Other & $6906(41.8)$ & & \\
\hline Multiple Visits to Index AD-diagnosing provider & $12,838(77.5)$ & & \\
\hline Only one Visit to Index AD-diagnosing provider & $3720(22.5)$ & & \\
\hline Time between first and second claim with AD diagnosis & $\begin{array}{l}\text { Average: } 92 \text { days } \\
\text { SD: } 125 \\
\text { Median: } 34 \text { days }\end{array}$ & & \\
\hline
\end{tabular}

Abbreviations: AD Alzheimer's disease, MAPD Medicare Advantage Prescription Drug, SD Standard deviation

Cohorts were matched on age, gender, race and length of pre-index enrollment

$¥$ Categories were merged; *Chi square test used for categorical variables; Wilcoxon rank sum test used for continuous variables; Significance level set at $P<0.05$

Please note that for identifying most commonly seen provider were identified prior to matching $(N=16,558)$ and we have identified only outpatient visits. The

index diagnoses for 35 patients were not at an outpatient facility. The specialties of $15.7 \%$ of the index AD-diagnosing providers were unknown because the

specialty data were missing from the claims database

comorbidities evaluated, hypertension, dyslipidemia, and other forms of heart disease were the most prevalent in both cohorts (Table 3). Other pre-specified comorbidities with notable differences each occurring more frequently in the $\mathrm{AD}$ cohort included mood disorders $(+$ $22.3 \%$ difference), cerebrovascular disease $(+20.5 \%$ difference), urinary tract infection $(+14.8 \%$ difference), anxiety disorder $(+11.4 \%$ difference $)$, and $\mathrm{MCI}(+10.5 \%$ difference).

When comparing the average pre-index HCRU (Fig. 2) the number of outpatient visits, hospitalizations, and ED visits at 6-month intervals were similar between the cohorts until six months prior to the index date. For the $\mathrm{AD}$ cohort, the number of these visits increased during the six months prior to the index date. A similar trend was observed for healthcare costs, with an increase in mean and median medical costs for the $\mathrm{AD}$ cohort occurring during the -6 -month interval prior to the index date (Fig. 3). During this time period, the average total healthcare cost per person was significantly higher for the $\mathrm{AD}$ cohort than for the matched cohort $(\$ 10,054$ vs. $\$ 4833, P<0.0001)$.

Using the CART analysis, up to 67 potential predictors of an $\mathrm{AD}$ diagnosis were evaluated, and the top 15 variables by importance were identified for inclusion in the logistic regression model (Fig. 4). From the tree, we see that use of $\mathrm{AD}$ medication is the most important predictor of an $\mathrm{AD}$ diagnosis and that an MCI or behavioral disturbance diagnosis, presence of an emergency department visit, and patient age provided additional information to classify patients as likely to receive an AD diagnosis. Due to its size, only the first part of the full tree is illustrated. The model showed excellent specificity (97\%) and acceptable sensitivity (77\%), and the overall area under the curve was 0.917 . The 15 top performing variables and their

Table 2 Clinical characteristics of Alzheimer's disease and matched cohorts

\begin{tabular}{|c|c|c|c|c|c|}
\hline & \multicolumn{2}{|c|}{$\mathrm{AD}(n=16,494)$} & \multicolumn{2}{|c|}{ Matched Cohort (No AD or Dementia) $(n=49,482)$} & \multirow[t]{2}{*}{$P$ value* } \\
\hline & Mean [SD] & $\overline{\text { Median }}$ & Mean [SD] & Median & \\
\hline Deyo Charlson Comorbidity Index & $3.8[3.0]$ & 3.0 & $3.0[2.9]$ & 2.0 & $<0.001$ \\
\hline RxRisk-V Score & $9.8[4.0]$ & 10.0 & $8.3[3.7]$ & 8.0 & $<0.001$ \\
\hline Number of Unique Medications Used (Drug Classes) & $22.0[14.0]$ & 20.0 & $18.2[10.7]$ & 17.0 & \\
\hline \multicolumn{6}{|l|}{ Wellness Visit and Assessments, n (\%) } \\
\hline Annual Medicare Wellness Visit & $4832(29.5)$ & & $17,132(35.0)$ & & $<0.001$ \\
\hline Cognitive Assessment & $435(2.6)$ & & $114(0.2)$ & & $<0.001$ \\
\hline AD medication use at baseline & $10,559(64.0)$ & & $884(1.8)$ & & \\
\hline
\end{tabular}

Abbreviations: $A D$ Alzheimer's disease, $M C I$ Mild Cognitive Impairment, $S D$ Standard deviation

RxRisk-V Score was calculated for those with at least one prescription

${ }^{*}$ Chi square test used for categorical variables; Wilcoxon rank sum test used for continuous variables; Significance level set at $P<0.05$ 
Table 3 Prevalence of pre-specified comorbidities for Alzheimer's disease and matched cohorts

\begin{tabular}{|c|c|c|c|}
\hline Comorbidity, n (\%) & $\mathrm{AD}(n=16,494)$ & $\begin{array}{l}\text { Matched Cohort (No AD or } \\
\text { Dementia) }(n=49,482)\end{array}$ & $P$ value $^{*}$ \\
\hline Hypertension & $14,816(89.8)$ & $43,198(87.4)$ & $<0.001$ \\
\hline Dyslipidemia & $13,951(84.6)$ & $41,824(84.6)$ & 0.986 \\
\hline Other forms of heart disease & $9434(57.2)$ & $23,597(47.7)$ & $<0.001$ \\
\hline Osteoarthritis & $8372(50.8)$ & $23,389(47.3)$ & $<0.001$ \\
\hline Urinary tract infection & $8146(49.4)$ & $17,096(34.6)$ & $<0.001$ \\
\hline Cerebrovascular disease & $7207(43.7)$ & $11,479(23.2)$ & $<0.001$ \\
\hline Ischemic heart disease & $6963(42.2)$ & $17,841(36.1)$ & $<0.001$ \\
\hline Diabetes & $6866(41.6)$ & $18,698(37.8)$ & $<0.001$ \\
\hline Mood disorder & $6483(39.3)$ & $8391(17.0)$ & $<0.001$ \\
\hline Osteoporosis & $5788(35.1)$ & $16,325(33.0)$ & $<0.001$ \\
\hline Any chronic obstructive pulmonary disease & $5728(34.7)$ & $15,405(31.2)$ & $<0.001$ \\
\hline Acute respiratory infection & $5137(31.1)$ & $16,576(33.5)$ & $<0.001$ \\
\hline Cancer & $4390(26.6)$ & $14,271(28.9)$ & $<0.001$ \\
\hline Anxiety disorder & $4356(26.4)$ & $7428(15.0)$ & $<0.001$ \\
\hline Heart failure & $3957(24.0)$ & $8907(18.0)$ & $<0.001$ \\
\hline Peripheral vascular disease & $3547(21.5)$ & $8155(16.5)$ & $<0.001$ \\
\hline Atherosclerosis & $3435(20.8)$ & $8615(17.4)$ & $<0.001$ \\
\hline Pneumonia & 2931(17.8) & $5506(11.1)$ & $<0.001$ \\
\hline Insomnia & 2132(12.9) & $4704(9.5)$ & $<0.001$ \\
\hline Mild Cognitive Impairment & $1727(10.5)$ & $0(0.0)$ & $<0.001$ \\
\hline Diseases of pulmonary circulation & $1689(10.2)$ & $4248(8.6)$ & $<0.001$ \\
\hline Chronic ulcer of skin & 1505(9.1) & $2486(5.0)$ & $<0.001$ \\
\hline Behavioral disturbance & 1369(8.3) & $0(0.0)$ & $<0.001$ \\
\hline Venous Thromboembolism & $969(5.9)$ & $1999(4.0)$ & $<0.001$ \\
\hline Parkinson's disease & $872(5.3)$ & $568(1.1)$ & $<0.001$ \\
\hline Gastric, duodenal, peptic, or gastrojejunal ulcer & $854(5.2)$ & $1710(3.5)$ & $<0.001$ \\
\hline Epilepsy & $845(5.1)$ & 688(1.4) & $<0.001$ \\
\hline Rheumatoid arthritis & $771(4.7)$ & $2155(4.4)$ & 0.087 \\
\hline Lung cancer & 233(1.4) & $720(1.5)$ & 0.686 \\
\hline
\end{tabular}

Abbreviations: $A D$ Alzheimer's disease, $M C I$ Mild Cognitive Impairment

${ }^{*}$ Chi square test used; Significance level set at $P<0.05$

interactions were used to run a logistic regression model (Fig. 5, Additional file 1: Table S1).

The results of the logistic regression analysis (Additional file 1: Table S1) showed AD medication use had the greatest association with increased likelihood of $\mathrm{AD}$ diagnosis $(\mathrm{OR}=161.157, P<0.001)$ followed by the presence of comorbidities such as MCI $(\mathrm{OR}=111.626, P<0.001)$, Parkinson's disease $(\mathrm{OR}=10.081, P<0.001)$, mood disorder $(\mathrm{OR}$ $=3.083, P<0.001)$, cerebrovascular disease $(\mathrm{OR}=1.856, P$ $=0.007$ ), and $/$ or a urinary tract infection (UTI, OR $=1.246$, $P<0.001)$ and ED visits $(\mathrm{OR}=1.131, P<0.001)$.

Although there were significant two-way interactions involving $\mathrm{AD}$ medication use, ED visit, and mood disorder, estimates at the mean values and distinct categories of interacting variables with these terms were consistently significant and greater than one. Similarly, odds ratios for Parkinson's disease were consistently greater than one and 5 of the 8 were statistically significant. Thus, the direction of main effects related to $\mathrm{AD}$ medication use, ED visit, mood disorder, and Parkinson's disease can be interpreted. There were no significant two-way interactions with MCI or UTI, facilitating the interpretation of these effects.

The main effect of cerebrovascular disease is not interpretable due to significant two-way interactions and odds ratios that varied in direction. Specifically, the presence of a cerebrovascular disease diagnosis indicated lower likelihood of an AD diagnosis in patients with a cognitive assessment as part of the annual wellness visit (3 of 4 odds ratios significant). Conversely, among 


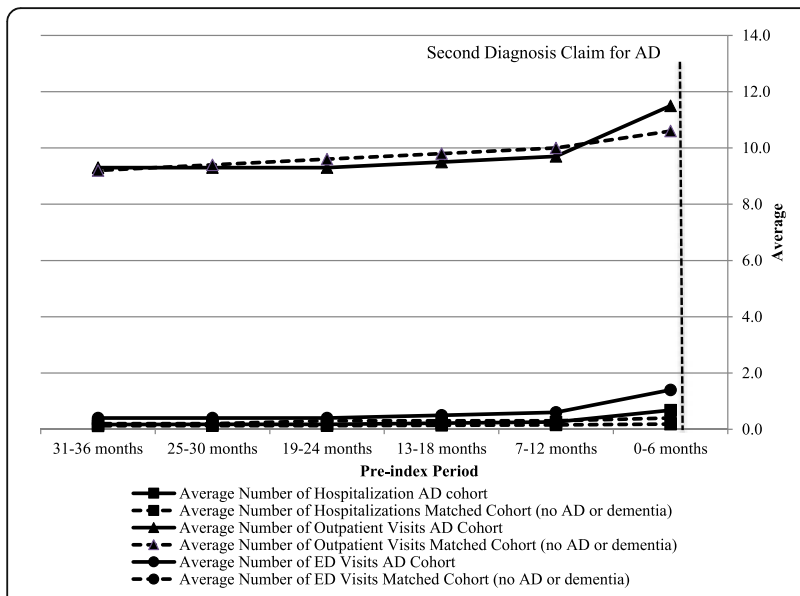

Fig. 2 Trends in healthcare resource use per person at six-month intervals for $A D$ and matched cohorts. Abbreviations: AD-Alzheimer's disease, ED-Emergency department

patients who did not have a cognitive assessment as part of the annual wellness visit, the cerebrovascular diagnosis indicated a greater likelihood of AD diagnosis ( 3 of 4 odds ratios significant).

Increasing age $(\mathrm{OR}=0.937, P<0.001)$ decreased the odds of being diagnosed with $\mathrm{AD}$, but this finding should

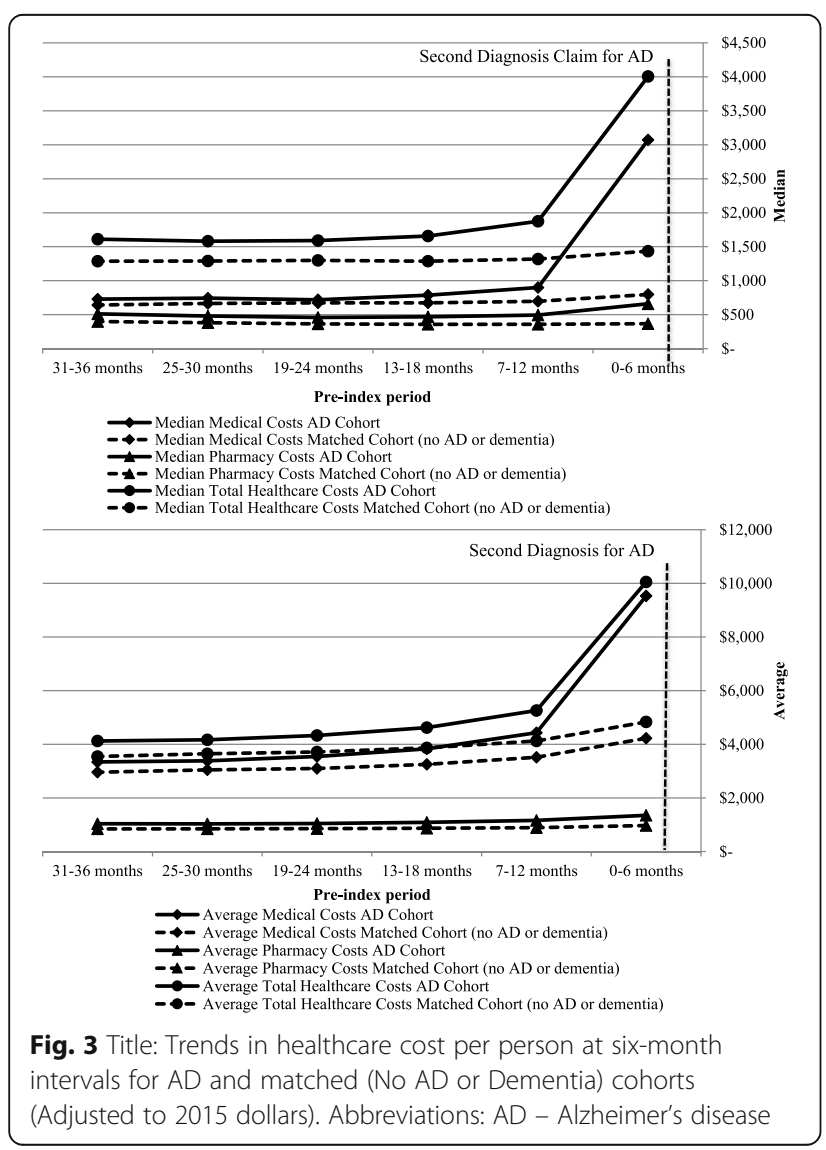

be interpreted with caution as the $\mathrm{AD}$ and matched cohorts were matched on age. Additionally, there were significant two way interactions between age and Parkinson's disease, and between age and cerebrovascular disease. When examining the 96 odds ratios involving age and combinations of values for each of AD Medication use, mood disorder, cerebrovascular disorder, Parkinson's disease, and income, the majority are significant, 71 were less than one, indicating increased age was associated with lower likelihood of AD and 6 were less than 1. The patient subgroups in which increased age was associated with greater likelihood of $\mathrm{AD}$ were those who had not received $\mathrm{AD}$ medications and did not have a diagnosis of mood disorder, cerebrovascular disease or Parkinson's disease and this OR was significant in four of the 6 income categories. The other subgroups in which increased age was associated with greater likelihood of $\mathrm{AD}$ were those in the upper two income categories who had not received AD medications, did not have a diagnosis of mood disorder or Parkinson's disease but did have a cerebrovascular diagnosis.

\section{Discussion}

With our study's longer duration of time to observe patients' engagement with the healthcare system, we were able to add to the existing understanding of a patients' journey prior to confirmatory AD diagnosis. Our study results significantly add to existing literature as the sample of 16,494 patients with a confirmatory diagnosis of AD is notable in terms of its longevity; the median duration of the pre-index period for the $\mathrm{AD}$ and matched cohorts was 72 months. In contrast, similar studies by Lin et al. (2016) [11] observed patients 24 months before and after their AD diagnosis, and Gilden et al. (2015) [6] required 12 months of data prior to and following AD diagnosis.

Over a third of the sample received their confirmatory $\mathrm{AD}$ diagnosis in the primary care setting, in comparison to less than one fourth receiving this diagnosis from a geriatric or mental health specialist. Also, we found that almost two-thirds of the AD patients received AD-related medications prior to their confirmatory diagnosis, and only $10 \%$ of patients in the AD group received an MCI diagnosis prior to their confirmatory diagnosis, all of which may suggest a reluctance to diagnose memory related issues [19]. Some factors that may contribute to the reluctance to make a diagnosis of AD include physician's decision not to create or increase the emotional stress of a patient. Alternatively, reluctance to make a diagnosis of $\mathrm{AD}$ may be reflective of insufficient training or time to confidently make a diagnosis through appropriate assessments resulting in a delayed diagnosis, especially given the ongoing challenge to diagnose based on exclusions [19, 20]. Without a biomarker or pathognomonic test, the diagnosis is always going to be somewhat questionable. 


\section{Subtree Starting at Node $=0$}

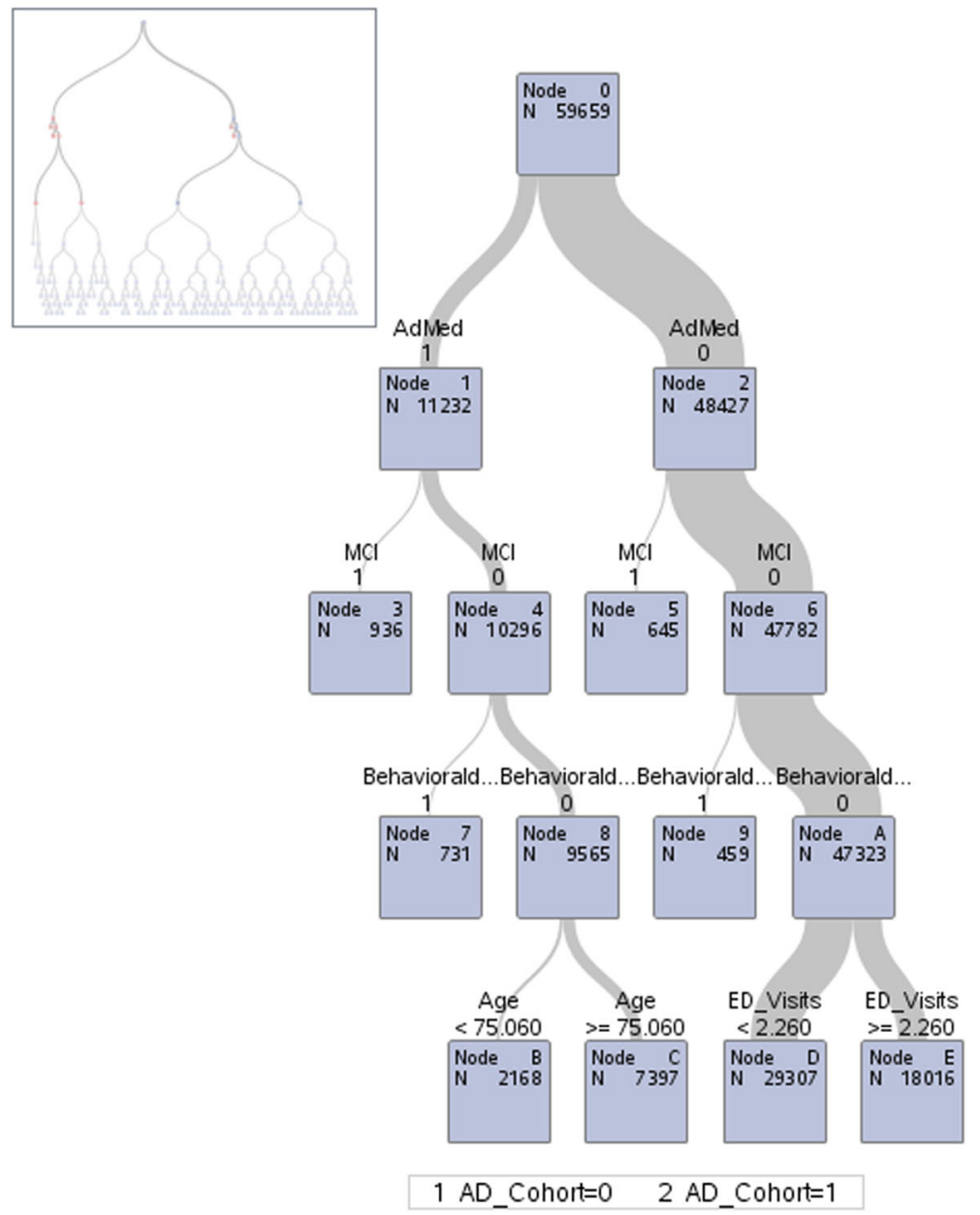

Fig. 4 Classification tree model sub-tree graph of factors most predictive of a diagnosis of AD. Abbreviations: AD - Alzheimer's disease, ED Emergency Department, $\mathrm{MCl}$ - Mild Cognitive Impairment; ADMed - AD medications, Behavioral - Behavioral disturbance Estimates from model controlling for behavioral segmentation.

The AD cohort was generally in poorer health than the matched cohort, which was reflected in the significantly higher comorbidity indices and greater odds of comorbid mental and behavioral diagnoses, cerebrovascular disease, and other diseases such as heart disease, UTIs, and pneumonia. This is a similar clinical profile to that observed in the post-index follow-up period by Suehs et al. (2013) [9], with exception of MCI and behavioral disturbance, which were not examined. The presence of various comorbidities in patients with $\mathrm{AD}$ highlights the importance that treatment plans for such patients should not be only focused on the expected cognitive decline, but should warrant a multidisciplinary approach to routinely assess for comorbid conditions.

Consistent with other administrative claims studies examining HCRU and cost in the year prior to the first AD diagnosis, $[8,9]$ this study showed that HCRU for patients with $\mathrm{AD}$ was significantly higher than that for the matched cohort. Similarly, Lin and others demonstrated that for patients with $\mathrm{AD}-\mathrm{RD}$, from the $5 \%$ Medicare sample, HCRU was greater than the control population during the 24 months prior to their first diagnosis, particularly during the most proximal 12 months [11]. This was driven by inpatient, home health, and post-acute care, with virtually no difference in outpatient or physician office visits. In contrast, a prospective study by Zhu et al (2015), [21] that included Medicare beneficiaries who received clinical evaluations for $\mathrm{AD}$ every 18 months, found significant differences only in home health and durable medical equipment use. Observed differences in HCRU in these studies could be due to case ascertainment (AD-RD, AD by clinical evaluation, or $\mathrm{AD}$ determined based on the presence of two ICD-9 codes), specific study population evaluated (MAPD, a multi-ethnic cohort from northern Manhattan, FFS Medicare), or changes in the approach to medical care of elderly patients over time. 


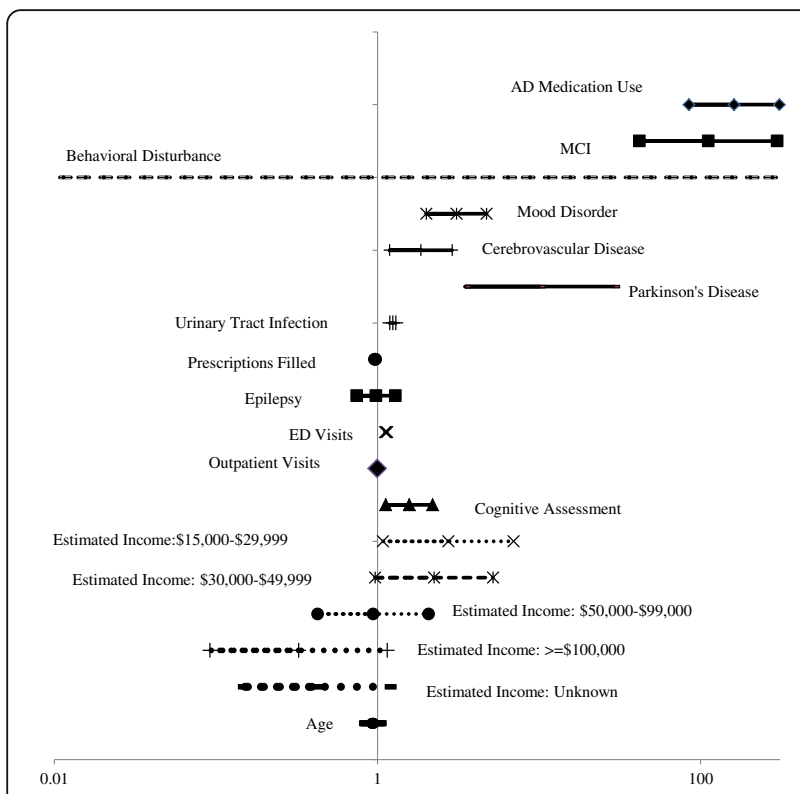

Fig. 5 Forest Plot for Logistic Regression: Demographic and Clinical Variables Associated with Diagnosis of AD. Abbreviations: AD - Alzheimer's disease, ED - Emergency Department, MCl Mild Cognitive Impairment Estimates from model controlling for behavioral segmentation. The left and right symbol are the lower and upper limits of the 95\% confidence interval for the odds of a diagnosis of Alzheimer's disease

In our study, the total average cost for patients at six months prior to AD diagnosis was greater (\$5221 in 2015 dollars) than for the matched cohort. One plausible explanation for this difference may be related to the comorbidity burden. Matching between the AD cohort and the non-AD or dementia cohort was not based on comorbidity, which allowed for a better understanding of the relative comorbidity differences across the cohorts. Additionally, costs associated with the subsequent AD diagnosis are also contributory to the difference. In comparison, the study by Lin et al. identified individuals on the basis of their first recorded AD-RD diagnosis code and found the difference in average pre-diagnosis costs in the six months prior to index was \$3571 (in 2014 dollars) [11]. The comparison of AD-RD to control subjects in the $5 \%$ Medicare sample included comorbidity in the identification of the matched cohort, supporting the premise that additional costs are incurred as a result of a subsequent AD diagnosis [11].

Our analysis of examining potential predictors of $\mathrm{AD}$ is similar to the findings of the study by Jaakkimainen et al. (2016), [5] which showed that the best performing algorithm in Canadian administrative claims data from primary care practices was either a hospitalization or three physician claims separated by 30 days or more in the same 24-month period. The sensitivity and positive predictive value of the algorithm was improved by inclusion of a prescription for a cholinesterase inhibitor. In the CART model, which had good sensitivity and higher specificity, AD medication use, diagnosis of MCI, Parkinson's disease, and a mood disorder were the strongest predictors of an AD diagnosis.

In extending the interpretation of $\mathrm{AD}$ medication use by exploring their interactions with other predictors, we found that the magnitude of association of $\mathrm{AD}$ medication use with an $\mathrm{AD}$ diagnosis varied, depending on whether patients also had cerebrovascular disease, Parkinson's disease, mood disorder, or UTI. The odds of AD diagnosis associated with AD medication use was uniformly lower in the subgroups of patients with Parkinson's disease, UTI, mood disorder or cerebrovascular disease in comparison to patients who were similar on all variables but that particular diagnosis. The predictor of $\mathrm{AD}$ medication use may reflect physician and/or patient preference to treat with symptomatic medication prior to receiving a definitive diagnosis, [6] while the most important psychiatric and neurological diagnoses likely reflect disease progression.

The current study's findings should be interpreted in the light of the following limitations. Characteristic of retrospective, claims-based research, the results may have been influenced by missing data, potential errors in coding, and unmeasured factors, such as psychosocial variables and other clinical variables. In addition, there is an established, multi-factorial gap in diagnosis and observance of $\mathrm{AD}$ symptoms. Furthermore, the commonality of the identified comorbidities in the non-AD population may limit the ability to use these as screening measures for potential AD. This study focused on patients with a confirmatory diagnosis for $\mathrm{AD}$ and did not include undiagnosed patients or those with only one claim for AD diagnosis. The index date for this study was the confirmatory diagnosis of $\mathrm{AD}$, which meant the costs would include the assessment and other AD-related costs from the first diagnosis of AD. Despite this, our findings are consistent with that of Lin et al., who evaluated costs prior to diagnosis of $\mathrm{AD}$ [11]. Additionally, data in this study were obtained from a single health insurance company, and although Humana is a large national health plan with members from various geographic regions, the results may not be generalizable to the overall U.S. population, or to subpopulations within certain geographic regions of the U.S. Moreover, the results may not be generalizable to all Medicare populations due to differences in benefit structure of MAPD and non-MAPD health plans. The matching criteria used in the current study, as well as the method for selecting the 
AD cohort, may be considered as additional study limitations. We matched the population only on demographic characteristics and not clinical characteristics such as comorbidities. This was so that we can understand the differences in comorbidities between patients, however, these comorbidities may have contributed to increases in healthcare resource use and costs. Additionally, we used second date of claim for $\mathrm{AD}$ as index date as opposed to the first. Healthcare resource and costs since the first visit with a claim of $\mathrm{AD}$ may have contributed to the increases observed during the six months prior to the second claim with $\mathrm{AD}$. However, in some sensitivity analyses conducted (not shown), the results did not change when first claim for $\mathrm{AD}$ was set as index date.

As an administrative claims study, we are only able to associate healthcare resource use with different cohorts defined by diagnostic codes. In the current study, our intent was to understand the total healthcare resource use, so we did not limit to claims specifically related to AD. Other types of observational research that include reason for cost are needed to understand if these increased costs are due to $\mathrm{AD}$.

\section{Conclusion}

Given the growing elderly population and the concomitant increases in prevalence of AD and associated cost of care, it is important to understand the patients' journey to diagnosis of this disease.

This study demonstrated that prior to the diagnosis of $\mathrm{AD}$, patients had a higher number of comorbidities and incurred higher costs in comparison to a demographically matched cohort. Certain comorbidities that occurred at a higher rate in the $\mathrm{AD}$ cohort, namely psychiatric and neurological in nature, may serve as flags to help identify patients most likely to develop $\mathrm{AD}$ using administrative claims data. We observed a trend of increasing healthcare costs during the 6-month period prior to the confirmatory diagnosis of $\mathrm{AD}$, offering yet another potential signal that can be gleaned from administrative claims data.

Without a biomarker or a test to detect AD, diagnosis is challenging, since it is essentially a diagnosis of exclusion. This suggests that $\mathrm{AD}$ is underdiagnosed, and the true impact of this disease may be greater than what the current study reports. With the pipeline of AD drug development aimed at disease modification, additional research is needed to further understand other clinical presentations that might potentially predict diagnosis of $\mathrm{AD}$ more robustly or even earlier than the 36-month-year period used in this study. Furthermore, with early recognition of $\mathrm{AD}$, a better understanding of the stage of illness at which patients are diagnosed with $\mathrm{AD}$, along with these early predictors, can help guide the treatment pathway.

\section{Additional file}

Additional file 1: Table S1. Logistic regression model of factors associated with the diagnoses of $A D$, based on classification tree model. This table lists the parameter estimates, $p$-values, odds ratios, and confidence intervals associated with the logistic regression model based on factors identified in the classification and regression tree analysis. (DOCX $80 \mathrm{~kb}$ )

\section{Abbreviations \\ AD: Alzheimer's Disease; AD-RD: Alzheimer's Disease and Related Dementias; CART: Classification and Regression Tree; DCCl: Deyo Charlson Comorbidity Index; ED: Emergency Department; FFS: Medicare Fee for Service; HCRU: Healthcare Resource Utilization; ICD-9-CM: International Statistical Classification of Diseases, Ninth Revision, Clinical Modification; \\ KBM: Knowledge Base Management; MAPD: Medicare Advantage with Prescription Drug Coverage; MCl: Mild Cognitive Impairment; NMDA: N- methyl-D-aspartate; RORs: Ratios of Odds Ratios; SD: Standard Deviation; UTI: Urinary Tract Infection}

\section{Acknowledgements}

The authors would like to acknowledge Mary Costantino, PhD for her editorial support.

\section{Funding}

This study was funded by Eli Lilly and Company. Employees of Eli Lilly and Company who coauthored this manuscript collaborated in the design of the study, in the interpretation of data, and in writing the manuscript.

\section{Availability of data and materials}

The data that support the findings of this study are available in aggregate form from Humana Inc. but restrictions apply to the availability of these data and individual level data are not publicly available. Inquiries for the data may be made to the authors.

\section{Authors' contributions}

Authors RN, VH, MS, NP, AF, DVA, MW, AD, LHF, VS, and DB were involved in the study design and interpretation. VS extracted data and MS analyzed the data. $\mathrm{RN}$ and $\mathrm{VH}$ were major contributors in writing the manuscript. Authors $\mathrm{RN}, \mathrm{VH}, \mathrm{MS}, \mathrm{NP}, \mathrm{AF}, \mathrm{DVA}, \mathrm{MW}, \mathrm{AD}, \mathrm{LHF}, \mathrm{VS}$, and DB reviewed, provided significant contributions and approved the final manuscript.

Ethics approval and consent to participate

This study was reviewed and received approval from Schulman IRB.

\section{Consent for publication}

Not applicable.

Competing interests

- Virginia S. Haynes, Adam S. Fleisher, Michael Witte, AnnCatherine Downing, are full-time employees and stockholders of Eli Lilly and Company.

Daniel E. Ball is a stockholder of Eli Lilly and Company.

- Radhika Nair and Nick Patel are employees of Comprehensive Health Insights, Humana Inc., which received funding and Sponsorship from Eli Lilly and Humana to conduct this study.

- Vishal Saundankar, Mir Siadaty, and Leslie Hazel-Fernandez, were employees of Comprehensive Health Insights, Humana Inc. at the time of the study.

- Daniel E. Ball was an employee of Eli Lilly and Company at the time of the study.

\section{Publisher's Note}

Springer Nature remains neutral with regard to jurisdictional claims in published maps and institutional affiliations. 


\section{Author details}

'Comprehensive Health Insights, Louisville, USA. 'Eli Lilly and Company, Indianapolis, USA. ${ }^{3}$ Humana Inc., Louisville, USA. ${ }^{4}$ Lilly Corporate Center, Drop Code 1730, Indianapolis, IN 46285, USA.

Received: 19 October 2017 Accepted: 16 September 2018

Published online: 16 October 2018

\section{References}

1. 2016 Alzheimer's disease facts and figures. Alzheimer's \& dementia : the journal of the Alzheimer's Association 2016, 12(4):459-509.

2. Dubois B, Feldman HH, Jacova C, Cummings JL, Dekosky ST, BarbergerGateau P, Delacourte A, Frisoni G, Fox NC, Galasko D, et al. Revising the definition of Alzheimer's disease: a new lexicon. The Lancet Neurology. 2010;9(11):1118-27.

3. Sperling RA, Aisen PS, Beckett LA, Bennett DA, Craft S, Fagan AM, Iwatsubo $T$, Jack CR Jr, Kaye J, Montine TJ, et al. Toward defining the preclinical stages of Alzheimer's disease: recommendations from the National Institute on Aging-Alzheimer's Association workgroups on diagnostic guidelines for Alzheimer's disease. Alzheimer's \& dementia : the journal of the Alzheimer's Association. 2011;7(3):280-92.

4. Roberts RO, Geda YE, Knopman DS, Cha RH, Pankratz VS, Boeve BF, Tangalos EG, Ivnik RJ, Rocca WA, Petersen RC. The incidence of $\mathrm{MCl}$ differs by subtype and is higher in men: the Mayo Clinic study of aging. Neurology. 2012;78(5):342-51.

5. Jaakkimainen RL, Bronskill SE, Tierney MC, Herrmann N, Green D, Young J, Ivers N, Butt D, Widdifield J, Tu K. Identification of physician-diagnosed Alzheimer's disease and related dementias in population-based administrative data: a validation study using family physicians' electronic medical records. Journal of Alzheimer's disease: JAD. 2016;54(1):337-49.

6. Gilden DM, Kubisiak JM, Sarsour K, Hunter CA. Diagnostic pathways to Alzheimer disease: costs incurred in a Medicare population. Alzheimer Dis Assoc Disord. 2015;29(4):330-7.

7. Geldmacher DS, Kirson NY, Birnbaum HG, Eapen S, Kantor E, Cummings AK, Joish VN. Pre-diagnosis excess acute care costs in Alzheimer's patients among a US Medicaid population. Applied health economics and health policy. 2013;11(4):407-13.

8. Frytak JR, Henk HJ, Zhao Y, Bowman L, Flynn JA, Nelson M. Health service utilization among Alzheimer's disease patients: evidence from managed care. Alzheimer's \& dementia : the journal of the Alzheimer's Association. 2008:4(5):361-7.

9. Suehs BT, Davis CD, Alvir J, van Amerongen D, Patel NC, Joshi AV, Faison WE, Shah SN. The clinical and economic burden of newly diagnosed Alzheimer's disease in a medicare advantage population. American journal of Alzheimer's disease and other dementias. 2013;28(4):384-92.

10. Suehs BT, Shah SN, Davis CD, Alvir J, Faison WE, Patel NC, van Amerongen D, Bobula J. Household members of persons with Alzheimer's disease: health conditions, healthcare resource use, and healthcare costs. J Am Geriatr Soc. 2014;62(3):435-41.

11. Lin PJ, Zhong Y, Fillit HM, Chen E, Neumann PJ. Medicare expenditures of individuals with Alzheimer's disease and related dementias or mild cognitive impairment before and after diagnosis. J Am Geriatr Soc. 2016; 64(8):1549-57.

12. What is Medicare Advantage. [https://www.medicare.gov/Pubs/pdf/11474.pdf]

13. Deyo RA, Cherkin DC, Ciol MA. Adapting a clinical comorbidity index for use with ICD-9-CM administrative databases. J Clin Epidemiol. 1992;45(6):613-9.

14. Klabunde CN, Potosky AL, Legler JM, Warren JL. Development of a comorbidity index using physician claims data. J Clin Epidemiol. 2000;53(12): 1258-67.

15. Quan H, Parsons GA, Ghali WA. Validity of information on comorbidity derived from ICD-9-CCM administrative data. Med Care. 2002;40(8):675-85.

16. Sloan KL, Sales AE, Liu CF, Fishman P, Nichol P, Suzuki NT, Sharp ND. Construction and characteristics of the RxRisk-V: a VA-adapted pharmacybased case-mix instrument. Med Care. 2003;41(6):761-74

17. Faries DE, Chen Y, Lipkovich I, Zagar A, Liu X, Obenchain RL. Local control for identifying subgroups of interest in observational research: persistence of treatment for major depressive disorder. Int J Methods Psychiatr Res. 2013;22(3):185-94.

18. Morgan J. Classification and regression tree analysis (technical report 1). In.: Boston University School of Public Health. Department of Health Policy \& Management.
19. Lang L, Clifford A, Wei L, Zhang D, Leung D, Augustine G, Danat IM, Zhou W, Copeland JR, Anstey KJ, et al. Prevalence and determinants of undetected dementia in the community: a systematic literature review and a meta-analysis. BMJ Open. 2017;7(2):e011146.

20. Dubois B, Padovani A, Scheltens P, Rossi A, Dell'Agnello G. Timely diagnosis for Alzheimer's disease: a literature review on benefits and challenges. Journal of Alzheimer's disease : JAD. 2016;49(3):617-31.

21. Zhu CW, Cosentino S, Ornstein K, Gu Y, Scarmeas N, Andrews H, Stern Y. Medicare utilization and expenditures around incident dementia in a multiethnic cohort. J Gerontol A Biol Sci Med Sci. 2015;70(11):1448-53.
Ready to submit your research? Choose BMC and benefit from:

- fast, convenient online submission

- thorough peer review by experienced researchers in your field

- rapid publication on acceptance

- support for research data, including large and complex data types

- gold Open Access which fosters wider collaboration and increased citations

- maximum visibility for your research: over $100 \mathrm{M}$ website views per year

At BMC, research is always in progress.

Learn more biomedcentral.com/submissions 